Ebisu

Études japonaises

Études japonaises

47 | printemps-été 2012

Catastrophes du 11 mars 2011, désastre de

Fukushima : fractures et émergences

\title{
Catastrophe environnementale au Japon : apport des savoirs profanes et mouvements citoyens
}

Environmental Disaster in Japan: Responding with Citizens Movements and Workaday Knowledge

日本における「環境炎害」一非専門的知識から市民運動一

Jean Lagane

\section{OpenEdition}

Journals

Édition électronique

URL : https://journals.openedition.org/ebisu/375

DOI : 10.4000/ebisu.375

ISSN : 2189-1893

Éditeur

Institut français de recherche sur le Japon à la Maison franco-japonaise (UMIFRE 19 MEAE-CNRS)

Édition imprimée

Date de publication : 1 juin 2012

Pagination : 143-150

ISSN : $1340-3656$

\section{Référence électronique}

Jean Lagane, « Catastrophe environnementale au Japon : apport des savoirs profanes et mouvements citoyens », Ebisu [En ligne], 47 | printemps-été 2012, mis en ligne le 03 avril 2014 consulté le 28 juin 2022. URL : http://journals.openedition.org/ebisu/375 ; DOI : https://doi.org/ 10.4000/ebisu.375 


\title{
Catastrophe environnementale au Japon Apport des savoirs profanes et mouvements citoyens
}

\author{
Jean LAGANE
}

La catastrophe du Tōhoku survenue le 11 mars 2011 rappelle brutalement le fait que territoires et sociétés sont confrontés à l'urgence de la question $\mathrm{du}$ respect de l'environnement et du " vivre ensemble». Cette contribution fait état des actions de mouvements citoyens particularistes au Japon et analyse la façon dont ces mobilisations participent de pratiques de résilience face aux catastrophes. En d'autres termes, il s'agit d'interroger la capacité de certaines communautés à absorber, à travers l'expression de savoirs profanes, des phénomènes de rupture. À ce titre, les catastrophes de Minamata 水俣 et de Toyama 富山 serviront d'exemples.

\section{Inégalités écologiques à Minamata et à Toyama et amorces de mobilisation}

Les questions vives liées à l'appréhension, l'analyse et la gestion des risques et des catastrophes interpellent de plus en plus la communauté de chercheurs en sciences humaines et sociales. Ulrich Beck (1992) constate l'inégalité cognitive et sociale des capacités de défense des populations sinistrées. Ces dernières peuvent recourir soit à " des conduites d'évitement et/ou d'autoprotection [...], soit à des mobilisations politiques pour obtenir des

\ Jean Lagane est enseignant-chercheur à l'université d'Aix-Marseille et membre du LAMES, Laboratoire méditerranéen de sociologie. Ses recherches portent sur la communication et la sociologie environnementales. 
autorités une amélioration de l'environnement» (Charles et al. 2007). Or, les populations les plus vulnérables socialement et économiquement acceptent plus volontiers de demeurer dans des environnements dégradés, ce qui facilite l'implantation "d'installations à risques qui peuvent continuer à polluer impunément " (Émelianoff 2007). Un ensemble de productions scientifiques dans le champ de la sociologie de l'environnement pointe une absence de justice environnementale. Ces travaux mettent en scène une inégalité des capacités de défense des populations face aux risques. Elvers, Gross et Heinrichs (2008) parlent « d'une intense hétérogénéité des sujets liée à la répartition des nuisances et des aménités environnementales tant à l'échelle locale qu'à l'échelle régionale ". Introduite au Royaume-Uni et en Allemagne de haut en bas, la justice environnementale s'est exprimée par la mise en place de politiques publiques. En revanche, au Japon comme aux États-Unis ${ }^{1}$, ce sont les mouvements civiques et autres parties prenantes (stakeholders) qui en ont été les ferments.

La maladie de Minamata et l'effervescence des études qui lui sont dédiées illustrent les dérives de l'idéologie du progrès social lors de ladite période de haute croissance au Japon (1955-1973). Elle est aujourd'hui reconnue comme l'épiphénomène des rejets mercuriels en milieu marin par l'usine Chisso $^{2}$ entre 1932 et 1966 près de la petite ville de pêcheurs de Minamata. Ce drame engendra des conséquences désastreuses (Minamata-byō 水俣 病) - névropathie, paralysie, urémie, malformations congénitales, décès. Il est estimé que " près de 2300 personnes ont été officiellement reconnues atteintes de la maladie de Minamata, plus de mille sont déjà mortes et plus de 17000 personnes ont demandé à être reconnues comme malades. » (Jobin $2006: 165$ ).

Les premiers cas de la maladie dite itai itai (itai itai byōイタイイタイ病, “j”ai mal, j'ai mal») - pathologie caractérisée par une perturbation du métabolisme et accompagnée de vives douleurs articulaires et osseuses - apparurent en 1946 chez des riverains de la rivière Jinzū 神通 à proximité de Toyama

1. La notion de justice environnementale est apparentée aux États-Unis, à la lutte contre la discrimination, face aux risques environnementaux, des classes défavorisées en raison de leur origine ethnique et/ou sociale. Ces situations d'inégalité renvoient à l'expression de « racisme environnemental " (Bullard 2005).

2. Il s'agit de l'usine électrochimique Nihon chisso hiryō kabushiki gaisha 日本窒素肥 料株式会社 qui fabriquait de l'acétaldéhyde. 
et firent de 1946 à 1968 une centaine de morts (Augier 2008 : 70-71). Des investigations mirent au jour le fait que « les malades consommaient du riz contaminé par l'eau d'arrosage captée dans la rivière qui recevait les effluents chargés en métaux ${ }^{3}$ de la mine de zinc de Kamioka " (ibid. : 71). Suite à des mesures effectuées en amont et en aval de la mine, des chercheurs démontrèrent que des sulfures de cadmium s'accumulaient dans les sédiments et contaminaient les rizières avant de migrer dans le riz, base alimentaire des riverains. L'aggravation de l'état de santé de ces derniers les exposait alors à une mort lente et douloureuse (Yamagata et Shigematsu 1970).

Les communautés sinistrées des pêcheurs de Minamata et des riziculteurs des villages longeant la rivière Jinzū, populations rurales et de niveau de formation intermédiaire, étaient fortement dépendantes en matière de pratiques alimentaires de l'excédent de leur production vivrière spécialisée. En outre, leurs positions modestes dans l'échelle sociale ne leur permettaient que difficilement de dénoncer les nuisances occasionnées par la contamination industrielle lors de la période de la haute croissance. Ainsi, ces deux cas de contamination industrielle de l'environnement ressortissent directement de la question de l'injustice environnementale. S'il est reconnu que le droit à l'information environnementale et aux contre-expertises s'inscrit en tant que paramètre clé de justice environnementale (Charles et al. 2007), ce droit ne s'imposa pas d'emblée au Japon et parfois plusieurs décennies furent nécessaires avant que les politiques publiques n'en prennent acte. Un exemple de contre-expertise fut soulevé dans le cas de Minamata. La pollution par le mercure fut d'abord déclarée comme pollution par le mercure non organique de l'usine Chisso. Il fallut attendre que des pathologies similaires apparaissent près de Niigata pour que le lien scientifique entre rejet de mercure organique en milieu marin et contamination dans la chaîne alimentaire soit officiellement reconnu, et vingt-deux années pour que le cadmium soit décrété responsable de la maladie itai itai (Augier $2008: 71$ ).

3. Et notamment en cadmium, sous-produit de l'extraction du zinc. 


\section{Des révoltes paysannes aux mobilisations environnementales}

Si la société japonaise renvoie l'image d'une société consensuelle, PierreFrançois Souyri rappelle qu'elle a néanmoins été le théâtre d'émeutes et de contestations répétées de l'autorité en place depuis le Moyen Âge. Le concept médiéval d'ikki 一揆, "alliance volontaire de personnes [...] en vue d'un objectif commun irréalisable avec des moyens ordinaires " (Souyri 1994 : 435-438), renvoie à des formes de " jacquerie ». L'historien poursuit en inscrivant ces coalitions paysannes et populaires dans un contexte plus large de bouleversement des hiérarchies, gekokujō 下攰上, « le monde à l'envers ». Ces mêmes mouvements prennent des formes et des répertoires d'actions collectives variés - du Mouvement pour la liberté et les droits du peuple (jiȳu minken undō 自由民権運動) de l'ère Meiji aux divers mouvements des années 1960-1970.

Les modalités de l'industrialisation nipponne, l'exiguïté des terres habitables et le phénomène croissant d'urbanisation, encouragés par le Deuxième Plan national d'aménagement du territoire ${ }^{4}$ adopté en 1969 et par le plan Tanaka de 1972, génèrent d'importantes nuisances industrielles et environnementales (kögai 公害) (Berque 1994: 171-172; Pelletier 1995 : 116-119).

Ainsi naissent des mouvements habitants " particularistes " de résistance (jümin undō 住民運動) (Berque 1994 : 172) face à cette industrialisation effrénée et à sa cohorte de nuisances dès la fin des années 1950 - engagements collectifs contre la ratification du traité de sécurité nippoaméricain (anpo hantai 安保反対), féministes, écologistes, voire de consommation engagée (teikei 提携) (Amemiya 2011, Lagane 2011).

Ces jümin undo $\bar{o}^{-5}$, renouant avec la tradition de l'ikki, vont augurer de la naissance des mouvements alternatifs de désobéissance civile caractéristiques

4. Ce plan d'aménagement a défini les contours de l'expansion industrielle de la période de haute croissance.

5. Ces mouvements sont liés à la défense d'intérêts concrets locaux et vont proliférer face à l'aggravation des nuisances environnementales jusqu'à atteindre près de dix mille mouvements citoyens en 1975 (Berque 1994 : 172). 
des sociétés occidentales de la fin du $\mathrm{Xx}^{e}$ siècle ${ }^{6}$. Peu à peu, ils se muent en mouvements de vigilance et de cogestion ou " mouvements citoyens » (shimin undō 市民運動) (Berque 1994 : 172). Les années soixante-dix suscitent une prise de conscience nationale avec la tenue des "Quatre grands procès de nuisances " - les affaires des cas de pollution industrielle de Yokkaichi, Minamata, Niigata et Toyama (McKean1981). La victoire des plaignants légitime ces mouvements citoyens avec la reconnaissance de la responsabilité de l'usine Chisso dans la pollution au mercure de la baie de Minamata. Le Japon, cherchant à effacer son cliché "d'animal économique " de la planète (Berque 1994 :172), entreprend alors une démarche publique de préservation de l'environnement - ameniti アメニテイ (aménité). À partir des années 1980 et sous la pression constante des mouvements citoyens, les recherches en sciences humaines et sociales au Japon traitent de plus en plus de phénomènes de nuisance environnementale. Ce renouveau engage les mouvements citoyens à structurer leurs actions dans une perspective socio-politique. Rajeunis sous l'action des furìtā フリーター7, ces mouvements s'organisent progressivement en tant que syndicats (rödō kumiai 労㗢 組合) au tournant du siècle et développent un nouveau répertoire d'actions collectives via l'usage des réseaux sociaux en tant qu'espace de solidarité et de revendication horizontales face aux autorités (Gaulène 2011).

\section{Reconnaissance de savoirs profanes et pratiques de résilience communautaire}

En guise d'illustration de l'opposition entre savoirs profanes et experts, nous limiterons notre démonstration à la présentation des recherches du sociologue britannique Brian Wynne. Ce dernier montra comment des éleveurs d'ovins du comté de Cumbrie, confrontés à des restrictions de pacage suite à des allégations de contamination radioactive après l'accident

6. Il est toutefois possible de faire remonter la notion de "désobéissance civile " (civil disobedience) au milieu du XIx ${ }^{\mathrm{e}}$ siècle (1849) à travers l'œuvre de Henry David Thoreau, essayiste et philosophe états-unien.

7. Les furìtā (freeters) sont des jeunes en emploi précaire en quête d'une nouvelle qualité de vie opposée aux contraintes de ladite classique entreprise nipponne fondée sur l'ancienneté et la séniorité. 
de Tchernobyl en 1986, enregistrèrent d'importantes pertes financières. Or, ces éleveurs avaient en vain tenté d'attirer l'attention des autorités sur l'usine locale de traitement nucléaire de Sellafield comme source potentielle de pollution. Par la suite, les experts se rallièrent à leur avis et décrétèrent que la radioactivité provenait bien de ce complexe nucléaire (Wynne 1992). Bien que des savoirs civils et profanes (grassroots knowledge) aient développé des formes intermédiaires d'expertise au sein des communautés rurales et se soient montrés sensibilisés aux formes de contrôle social - relations publiques - en matière de gestion des risques, ce cas demeure emblématique de la sous-estimation des risques environnementaux. Cet exemple fait écho à la place qu'occupent les collectifs citoyens japonais dans l'appréhension des catastrophes et à leur capacité d'organiser une action publique concertée. De tels usages s'inscrivent alors dans des pratiques de résilience ${ }^{8}$ de nature dynamique et évolutive qui allient une double capacité - résister et se reconstruire suite à un traumatisme (Tisseron 2009). De même, la résilience se traduit par les usages des nouveaux médias et des réseaux sociaux - courriels, SMS, forums, chats, blogs... pouvant amorcer des pratiques de résilience communautaire après des attaques terroristes (Fogel et Patino 2005). Ces mêmes modalités de résilience communautaire ont été interrogées, suite au passage de l'ouragan Katrina à la Nouvelle-Orléans, à travers l'analyse de leurs formes de capacité de subsistance des systèmes humains et techniques après un désastre naturel (Maret et Cadoul 2008). Elles ressemblent en bien des points aux usages japonais mentionnés précédemment.

\section{Conclusion}

Au-delà de la simple mise en relation de la triple catastrophe du 11 mars 2011 avec les contaminations industrielles de Minamata et de Toyama et de l'analyse des mobilisations civiles qui s'ensuivirent, cet article a permis d'appréhender et de déconstruire certains discours essentialistes, véhiculés par les médias sensationnalistes occidentaux, qui tendent à associer à l'image de la société japonaise les traits culturels d'une nation stoïque et résignée face aux désastres. En effet, il est possible de remettre en cause de telles opinions

8. Il faut cependant distinguer résiliences individuelle et collective. 
en raison des liens qui unissent l'essor de mouvements citoyens contemporains fondés sur la contestation à ceux de la tradition médiévale des coalitions paysannes. D'autre part, il apparaît que ces formes de mobilisation civile mettent au jour des potentiels de résilience communautaire qui valorisent les savoirs locaux et profanes et peuvent encourager le renouveau de politiques publiques favorables à la préservation de l'environnement et à la prévention des sinistres. En dépit de ces remarques teintées d'optimisme, on notera toutefois que, suite à la triple catastrophe du 11 mars 2011, la réalité des faits démontre une volonté persistante du gouvernement à encourager le retour des populations dans les zones fortement contaminées.

19 avril 2012

\section{Bibliographie}

AMEMIYA Hiroko (dir.), 2011

Du teikei aux AMAP, Rennes, PUR, Économie et société.

AUGIER Henry, 2008

«La mort à petit feu : le cadmium, la redoutable maladie itaï itaï », in AUGIER Henry (dir.), Le livre noir de l'environnement. État des lieux sur les pollutions planétaires, Paris, Alphée Jean-Claude Bertrand, pp. 70-71.

BECK Ulrich, 1992

Risk Society: Towards a New Modernity, London, Sage.

BERQUE Augustin, 1994

«L'environnement », in BERQUE

Augustin (dir.), Dictionnaire de la civilisation japonaise, Paris, Hazan, pp. 171-172.
BULLARD Robert D., 2005

The Quest for Environmental Justice:

Human Rights and the Politics of Pollution, San Francisco, Sierra Club Books.

ELVERS Horst-Dietrich, GROSS Matthias, HEINRICHS Harald, 2008

"The Diversity of Environmental Justice: Towards an European Approach ", European Societies, 10 (5) : 835-856.

ÉMELIANOFF Cyria, 2007

«La problématique des inégalités environnementales, un nouveau paysage conceptuel », Écologie et Politique, 35 : 19-31. 
FOGEL Jean-François

\& PATINO Bruno, 2005

Une presse sans Gutenberg, Paris, Grasset.

JOBIN Paul, 2006

Maladies industrielles et luttes syndicales au Japon, Paris, EHESS.

McKEAN Margaret A., 1981

Environmental Protest and Citizen

Politics in Japan, Berkeley, University of

California Press.

MARET Isabelle

\& CADOUL Thomas, 2008

«Résilience et reconstruction durable:

que nous apprend La Nouvelle-

Orléans ? ", Annales de géographie, mai, $663: 104-124$.

PELLETIER Philippe, 1995

«L'état de l'environnement » in

SABOURET Jean-François (dir.), L'état du

Japon, Paris, La Découverte, pp. 116-119.

SOUYRI Pierre-François, 1994

«Révoltes (mouvements sociaux) »

in BERQUE Augustin (dir.), Dictionnaire

de la civilisation japonaise, Farigliano/

Paris, Hazan, pp. 435-437.

TISSERON Serge, 2009

La résilience, Paris, PUF.

THOREAU Henry David, 1992

Walden; and, Resistance to Civil

Government: Authoritative Texts,

Thoreau's Journal, Reviews, and Essays in

Criticism, ROSSI William (éd.), New-York, Norton.
YAMAGATA Noboru

\& SHIGEMATSU Itsunari, 1970

"Cadmium Pollution in Perspective»,

Bulletin of the Institute of Public Health,

Tokyo, 19 : 1-27.

WYNNE Brian, 1992

«Misunderstood Misunderstanding:

Social Identities and Public Uptake

of Science ", Public Understanding of

Science, 1 : 281-304.

\section{Articles en ligne}

CHARLES Lionel, ÉMELIANOFF Cyria, GHORRA-GOBIN Cynthia, ROUSSEL Isabelle, ROUSSEL François-Xavier et SCARWELL Helga, 2007

"Les multiples facettes des inégalités écologiques ", Développement durable et territoires [en ligne], Dossier 9 : Inégalités écologiques, inégalités sociales, mis en ligne le 28 août 2007, consulté le 27 septembre 2011. URL : $<$ http://developpementdurable.revues. org/3892>.

\section{GAULÈNE Mathieu, 2011}

«L'autre Japon », [mercredi 06 avril 2011, 09 : 00]. URL : <http://www.nonfiction.fr/ article-4471-lautre_japon.htm $>$.

\section{LAGANE Jean, 2011}

« Du teikei à I'AMAP, un modèle acculturé », Développement durable et territoires [en ligne], mai, 2 (2), mis en ligne le 07 mai 2011. URL : <http:// developpementdurable.revues.org/ pdf8918>. 